\title{
The Development of Al in Medicine and the Research Environment of the SPHINX Project at the Start of the 1980s
}

\author{
M. Fieschi \\ LERTIM - Faculté de Médecine de Marseille, Aix-Marseille University, France
}

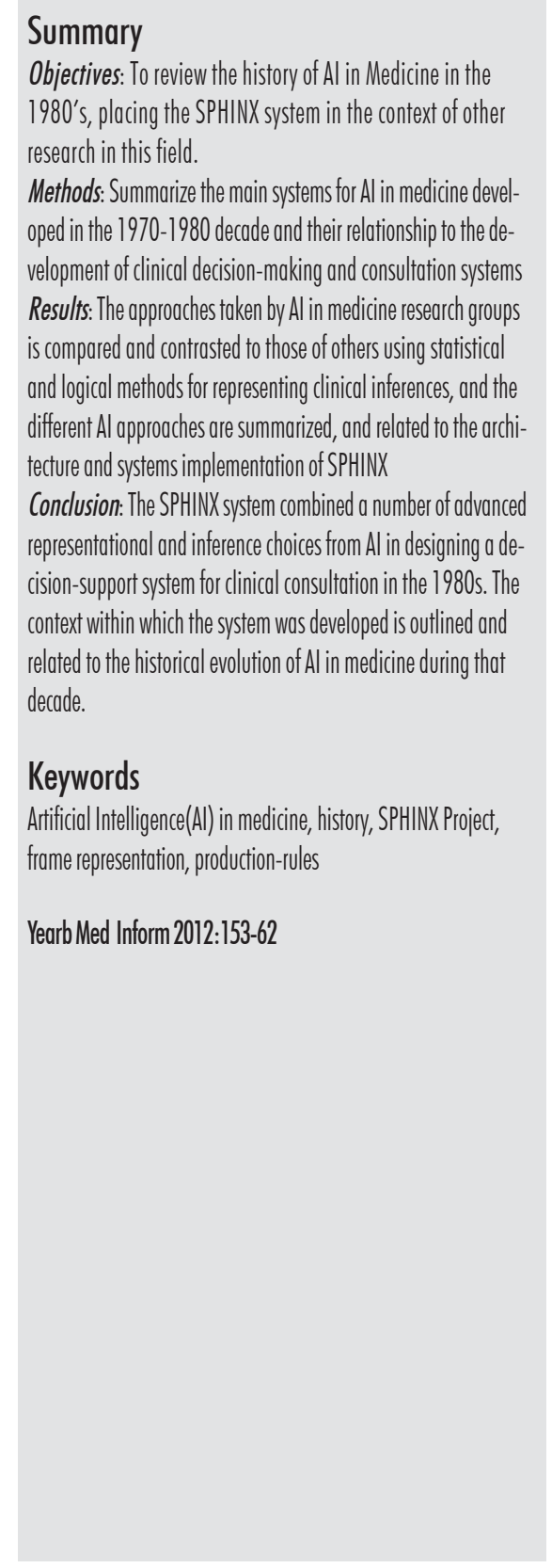

"The essence of science lies not in discovering new facts, but in discovering new ways of thinking about them »

W.L. Bragg, History of Science,1951

\section{Introduction}

The use of computers for medical applications began in the 1960s. Researchers have been attracted by the possibility of using computers to assist decision-making ever since access to them became possible. The emerging medical informatics community devoted considerable effort to the management of patient data and the aim of this pioneering research was generally to establish a good diagnosis based on all the information available in the patient's medical records. The technology available at the time was amenable to this approach. It should be borne in mind that only batch processing was possible, and the interactivity required to design a stepby-step decision-making tool defining the best strategy was well beyond reach during this early era. For this reason, computer use was disconnected from clinical activity in the strict sense of the word, and the goal of this research was to develop programs with performances comparable to those of doctors with expertise in the relevant clinical domain.

The researchers developed tools and techniques for modeling the clinical decision-making process. Theories based on algorithmic and numerical processing of information were among the most "naturally" used. Procedural treatment and the application of probability theory to assist diagnostic and therapeutic decision-making predominated. Many teams developed tools based on probabilities and Bayes' theorem. The pioneers in this field included Ledley and Lusted [1], Homer Warner [2], Gorry and Barnett [3] in the United States and Tim de Dombal [4] in Europe (UK).

These methods dominated the first decade, but they were not the only avenues of research followed by researchers, as shown in table 1. After the normative decision analysis centered on Bayes' rule, Artifical Intelligence (AI) approaches in the years 1975-1985 moved towards a symbolic approach to medical knowledge representation and heuristic methods for quantifying uncertainty.

It was in the context of the emerging development of AI that our team in Marseilles contributed to research into decision support and developed tools for use in this domain. SPHINX was one of the major projects on which we worked.

We will review these developments here, placing them in their historical context. As in all scientific disciplines, reviews of this type make it easier to appreciate the evolution of ideas and the ground covered. However, it is particularly important in an experimental discipline like ours that is strongly affected by the technological means available at a given moment for the development and refinement of paradigms. 
Table 1 Diagnostic methods adapted from Deutsch et al [5]

\begin{tabular}{|l|l|l|}
\hline Medical knowledge & Inference engine & Remarks \\
\hline Clinical cases & Classification tree & Followinstructions in an algorithm \\
Clinical cases & Pattern Recognition & Find a similar patient in the database \\
Neural net & Pattern Recognition & \\
$P(D), P(f / D)$, risks and utilities & Bayes and decision theory & Rank-order hypotheses \\
Discriminant function & Discriminant analysis & $\begin{array}{l}\text { Compute a posteriori probability of diseases } \\
\text { based on discriminant function }\end{array}$ \\
Belief measures & Demster Shafer Theory & $\begin{array}{l}\text { Compute degree of belief in single and } \\
\text { combined disorders }\end{array}$ \\
& Rule-base dinference & $\begin{array}{l}\text { Infer diseases with certainty factors or possibility } \\
\text { measures }\end{array}$ \\
Production rules & Criteria-base reasoning & Match findings to disease descriptions \\
Criteria table & Model-based reasoning & $\begin{array}{l}\text { Pathophysiological pathways from etiologies to } \\
\text { findings }\end{array}$ \\
Causal model of diseases & & \\
\end{tabular}

2 The Al Approach in Medicine: New Ways of Representing Knowledge and Providing Decision Support

The transformation of the field of AI led to the emergence of expert systems. The artificial intelligence or knowledge engineering approach was first developed in the mid 1970s and has since been applied as a methodology for computer-aided decision-making in medicine and many other fields. AI systems tackled the question of the use, and coherent and valid management, of large amounts of knowledge, including heuristic knowledge.

This approach and its results were highly credible. It launched entrepreneurial efforts and AI research projects in domains other than medicine, together with major initiatives, such as the Japanese Fifth-Generation plans, which had repercussions worldwide.

The potential value of these tools for use in clinical practice, based on their performance, was not the only motivation driving researchers. They were also interested in developing an analytical approach to medical reasoning and the analysis of non-probabilistic and less formalized, more cognitively real reasoning processes. It is both interesting and instructive to investigate other models, in terms of the less formal aspects of decision-making. Models were proposed for the representation of expert opinion and experts displayed a certain fascination with the analysis of their own inference rules. The abundance of publications during this era bears witness to the dynamic nature of the research carried out; it conveys the debates, arguments and importance of fundamental questions concerning reasoning in the presence of uncertainty and non-probabilistic and non-formalized reasoning using incomplete knowledge, the nature of the knowledge used, including metaknowledge and its representations.

Systems based on production rules provided a fundamental decoupling between control and rule-writing, facilitating the management of knowledge and its evolution (modifiability), theoretically making it possible to check the validity (consistency) of a knowledge base.

Two production rule-based systems thus profoundly marked this era [6] and our research:

- An approach focusing on the problem of cognitive psychology and attempts at modeling in this domain. This approach aimed to create programs implementing a theory of human behavior (including errors and omissions, for example) in the performance of simple tasks.[7]

- Knowledge-based expert systems (such as MYCIN, which was developed by EH Shortliffe [8] at Stanford University), which focused on performance. These systems were developed for the design and development of programs using production rules as a means of representing knowledge that had not previously been formalized, in a given domain, for the completion of tasks in a competent manner, making it possible to draw conclusions supported by various degrees of certainty or belief.

\section{Little-recognized Contributions from this Period of Expansion in Artificial Intelligence}

This was an incredibly dynamic time for research in this field, but, curiously, we tend to focus exclusively on the disappointments following this period, because the anticipated applications did not make it into the real world of clinical practice.

The detractors of these approaches often say that decision support systems cannot meet the challenge of medical practice in all its real complexity of daily routine application. It is true that if we consider only the number of systems used in practice, the results have been poor. There is a gap that has not been bridged: applications for use in everyday practice are lacking. However, in a more complete review, we must not forget the other contributions and benefits arising from research into decision support, which have been translated into medical practice in many ways. This research helped to rationalize medical acts, enriched the search for 
optimal strategies in terms of the definition of well-identified and defined explicit criteria, and provided frameworks for the analysis of decision-making processes, the knowledge used, the type of inference, justification of decisions and the search for better quality care at a lower cost (information and communication technologies have been identified as one of the means contributing to the improvements sought [9] [10] ). Finally, this work has made it possible to provide cognitive support in clinical situations (introduction of reminders and alerts, development of guidelines and of tools for implementing them etc.) and has encouraged reflections concerning the role of experts (evidence-based medicine).

We need to remember certain other advances from this period and this research that are even more specific to the AI approach. Here are a few examples:

- It was demonstrated that symbolic logic inference rules were a powerful alternative to the previously prevailing model, which was based exclusively on numerical (mostly probabilistic) methods.

- The simplicity of the syntax of production rules and the explicit relationships that they can establish between observations and medical concepts led to the analysis and then to the identification and use of semantics in the relationships based on these rules. This made it possible to distinguish and to use, in programs, associative relationships reflecting statistical correlations, causal relationships and taxonomic relationships (A is_a B). This has greatly enriched models and the reasoning capacity of systems and has paved the way for an approach based on the representation of explicit, rich knowledge, used, as it is today, in the construction and utilization of ontologies.

- Classically, information processing was only relevant if all the information relating to a problem had been collected, in an exhaustive manner, and all the configurations of data processing for this infor- mation had been identified. It rapidly became clear that only "simple" problems could be resolved by algorithmic processing. Problems based on knowledge are not of this type and it was in this context that AI was born and combined with classical algorithmic pathways for the exploration of other possibilities and to overcome the limitations of algorithm-based methods.

- AI has been proposed as an alternative to information science. According to Ganascia, it represents a break with a tradition inherited from information theory, it assigns an operating function to knowledge that is independent of its information content [11]. It has explored avenues outside the realm of classical algorithms and the standard representation of data. Semantic representations of data form the basis of what AI refers to as "knowledge representation", with the General Problem Solver (GPS) of Newell and Simon (1969) [12] being the first program to separate general resolution methods and specific knowledge.

- "Modularity" is another key characteristic of this approach. It is used for both knowledge representation and control, in metaknowledge for the use of this knowledge. The modularity of a program translates into the degree of separation of its functional units into potentially separable units. This is a fundamental issue in knowledge organization. Programs written in procedural languages essentially rely on procedures to ensure control between different sections of code. In 1975, Winograd [13] highlighted the value of production rules in his discussion of modularity in programming: "We can view production systems as a programming language in which all interaction is forced through a very narrow channel... and the formalism is interesting to the degree that complex processes can be described without resort to such kludgery, maintaining the clear modularity between the pieces of knowledge and the global process which uses them".

- All representations require formalization. Much AI research has focused on determining the most appropriate knowledge representations for achieving high performance from knowledge-based systems. The representation of concepts and complex objects has led to the development of models, such as Minsky's frames [14] (this mode of representation was used by Pauker and Szolovits [15] in the Present Illness Program (PIP) system). This research has triggered a general evolution of programming languages towards logical representations (Prolog) and then towards object-oriented languages, which are more suitable for the solution of this type of problem.

- From problem-solving to cognitive tasks: In the 1980s, some psychologists, computer engineers and researchers focused on solving a group of problems collectively referred to as man/machine interactions, which now form part of the field of ergonomics and cognitive ergonomics ${ }^{1}$. User behavior was analyzed in terms of cognitive science, leading to the development of the concept of a "user model".

\section{The Major Projects Contemporary to SPHINX, Developments in the USA and Europe}

We have already cited the work of $\mathrm{EH}$ Shortliffe and the Stanford group on the MYCIN project in the USA. This project gave rise to a family of systems,

Obviously, research on the application of informatics tools in medicine in the 1970s and early 1980s was based on very simple man/machine interfaces, with screens in character mode, an absence of graphical interfaces, with no mice, touchsensitive screens, portable computers and nomade devices and with other limitations 
including the VM system developed by L. Fagan [16] (1979) for monitoring artificial ventilation and the GUIDON and NEOMYCIN systems developed by W. Clancey for evaluating the educational potential of MYCIN [17].

On the East Coast, the group of P. Szolovits at MIT was developing the PIP system and the ABEL system [18], which makes use of a hierarchical representation of causal links for reasoning in the clinical domain of hydro-electrical disorders.

At Rutgers University (New Jersey), the team of C. Kulikowski [19] and S. Weiss developed, through CASNET, the use of a semantic network of causality for the diagnosis and treatment of glaucoma, and subsequently other diseases.

Other teams, of course, also worked on analogous projects, and although we cannot cite them all, we should mention the work of H. Pople [20], J. Myers and RA. Miller on the INTERNIST (in its early days known as DIALOG) project at the University of Pittsburg, Pennsylvania and that of PL. Miller [21] on the ATTENDING project at Yale University.

In Europe, interest in the new possibilities opened up by AI research in medicine was every bit as strong as in the US. We will mention some of the pioneers here, although the list is far from exhaustive.

In UK, Peter Hammond, at Imperial College London, developed the APEX Shell, and Alan Rector [22], at Manchester University, focused on primary care problems. Other groups, like that of P. Skonsen at St. Thomas Hospital, London, studied applications relating to diabetes treatment, and the group of E. Carson and D. Cramp [23] at the Royal Free Hospital University in London, worked on physiological modeling for problems in intensive care units. The group directed by John Fox [24] at the Imperical Cancer Research Foundation in London also made important contributions, working on several projects related to protein structure analyses, leukemia and the Oxford System of Medicine (OSM) project.

In Sweden, the group at Linkoping University run by $\mathrm{O}$. Wigerz and $\mathrm{T}$. Timpka [25] worked on primary healthcare and the conditions in which expert systems could be applied in this domain (constraints on use, environmental constraints, additional knowledge).

In German-speaking countries, the group of P. Reichertz from Hanover and the CADIAG system developed by K. Adlassnig [26] using fuzzy set theory were also of particular importance.

In Italy, the highly active group of G. Molino in Turin [27] developed an expert system for the evaluation of liver function. However, the most active group in this domain was that directed by Mario Stefanelli at the University of Pavia, which launched this research activity with the ANEMIA system [28] developed using the EXPERT system rule-based shell [29] produced by Weiss and Kulikowski at Rutgers University.

In France, there were five main university hospital groups working in the field of AI. They were located in Grenoble, Lille, Marseilles, Montpellier and Paris. These five teams received funding from the French Ministry of Health and from the Centre Mondial de l'Informatique (the World Computing Center) in 1984. The funding program provided the five teams with the same computing equipment, machines from Digital Equipment Corporation (Vax 11-780 machines, which replaced the PDP-11 line) and funding for systems engineers.

The Parisian team, led by O. Gascuel, developed the SAM system [30], which used production rules to represent knowledge and a model of reasoning in the presence of uncertainty similar to that of MYCIN, for applications in the treatment of arterial hypertension and the diagnosis of abdominal pain.

The team in Lille used, in a first version, the inference motor developed by $\mathrm{O}$. Gascuel to develop applications in the domains of diabetes treatment and antibiotic use [31]. This project was carried out in collaboration with the team in Grenoble. The team from Montpellier initiated an ONCOCINlike project in the domain of lung cancer treatment.

We will round off this rapid review with the work of our Marseilles team from 1978 to 1987. Medical decision support was a key area of research for our team, which worked on different formalisms for knowledge representation, the use of logic-based models in decision support and reasoning in the presence of uncertainty. We ran many projects in addition to SPHINX, which we will present in more detail. These projects concerned theoretical work on reasoning in the presence of uncertainty, particularly fuzzy subsets, under the direction of E. Sanchez [32], and also considered clinical applications. Two of these projects were of particular importance:

- The GENDIAG project, led by J. Gouvernet [33], concerned an application for the diagnosis of birth defects. It was based on a hierarchical representation of knowledge and implemented Schafer's theory for the weighting of hypotheses. It included and could identify 400 genetic syndromes.

- The PROTIS system, developed by G. Soula [34], dealt with diabetes treatment through the implementation of decision rules and a model of reasoning in the presence of uncertainty based on fuzzy subset theory.

\section{The Birth of Artifical Intelligence in Medicine Europe (AIME) from Pioneering Initiatives}

Many contacts had been established with numerous leaders in the field from the United States. This explains the development strategies in this area of research, which were often based on tools from across the Atlantic (as at Pavia) or were at least strongly inspired by such tools. These contacts led to the organization of 
scientific meetings and seminars, attended by our American colleagues, together with exchanges and collaborations. Thus, C. Kulikowski participated at conferences on reasoning in the presence of uncertainty and fuzzy subsets organized by our team at Marseille, and on AI in medicine at Pavia in 1985. These contacts also resulted in several collaborations, including that between the Parisian team and P. Szolovits, with P. Szolovits acting as an examiner for my $\mathrm{PhD}$ thesis in Marseilles. It was also through these contacts that I was invited to work as a visiting scientist in the laboratory of P. Szolovits at MIT.

In September 1985, Mario Stefanelli organized a first international conference on AI in medicine at Pavia. Following this conference, we organized a meeting at Marseilles in June 1986, at which the decision was taken to set up a European Society for Artificial Intelligence in Medicine (AIME). It was under the auspices of this scientific society that we organized a first colloquium bearing the name "Artificial Intelligence in Medicine - Europe (AIME)" at Marseilles in August/September 1987. This meeting brought together 180 researchers and the proceedings, edited by J. Fox, M. Fieschi and R. Engelbrecht, were published in the Lecture Notes in Medical Informatics series by Springer. JH. Van Bemmel ran the inaugural conference and L. Fagan was the keynote speaker of this first European colloquium. AIME has since organized a colloquium of this type every two years.

European Commission calls for proposals have led to the establishment of collaborations between many different teams working in this domain. The projects concerned have included DIABETA and GAMES [35], both of which involved our team.

\section{The SPHINX Project}

This project was based on my $\mathrm{PhD}$ work. Masson (publisher, Paris) published a version of this thesis [36], in- cluding additional material, in 1984. In 1986 and 1987, they also published translations in Italian and Spanish. Chapman and Hall have published an English version [37]. The project was supported by grants from the CNRS and the Agence de l'Informatique (ADI). The aim was to develop a model and a shell independent of applications, and to provide development and knowledge-base management tools suitable for use by users without specialist computing skills.

This project provided us with an opportunity to investigate important questions for the development of consulting systems with "intelligent behavior" likely to supply pertinent advice. We developed a knowledge representation model to ensure the consistency (common sense) and expertise relevant to the medical approach (diagnostic or therapeutic). We also worked on the notion of context. We aimed to demonstrate the importance of the description of a situation for the development of an effective interface with the user and implementation of the relevant parts of the available information. This description of context was a heuristic feature of our model and was based on knowledge expressed with uncertainty. Most of the systems at the time made use of rules with either forward or backward chaining. We developed a tool with which it was possible to work with either data-driven or goal-directed strategies, depending on the phase of a consultation and the type of knowledge used in it.

The main aim of the SPHINX project [38] was to provide clinicians with a consultant system aid to diagnostic, therapeutic or preventive decision-making, with four basic objectives:

1. The knowledge should lead the system to give advice comparable to that of an expert.

2. Dialog with the machine should be conducted in a language as clear and as close as possible to natural language to avoid the need for the user to learn a particular language or code.

3. The system should potentially be able to explain its decisions by providing the elements on which its reasoning is based.

4. The system should be able to improve its advice, to adapt to changes in medical data and to increase its applications through a data acquisition module that is simple to use for the layman.

Two domains of application were selected: the diagnosis of jaundice and therapeutic decision support for non insulin-dependent diabetes.

No appropriate language interpreter or compiler was available at the start of the project. We therefore used Fortran IV for program development.

According to the prevailing paradigm, consulting systems were designed based on "expertise". In other words, knowledge engineering was a prime example of maieutics, in which the aim was to get experts in the domain to make knowledge rules explicit (evidencebased medicine and good practice guidelines had yet to emerge).

The construction of a consulting system making use of expert knowledge implicitly involved searching for a system that displayed behavior most like that of a human expert. Just as a doctor listens to his patient to understand the reasons for the patient's visit, so the system should be able to accept data supplied spontaneously by the user and to ask for more information, because the data already in the system recalls a particular diagnosis or a number of possible diagnoses. With this approach, it is important to direct the search for, and use of, relevant data.

At the early stage of the consultation the system uses heuristic-oriented knowledge, which we called the "evocation power" of the recall-signs; that is, it uses knowledge and approximate reasoning which can be modelled on the theory of fuzzy sets, many valued logic [39] or credibility functions de Shafer [40]. We have chosen to apply Soula's use of fuzzy logic [41] on the extended Modus Ponens and extended Modus Tollens to many valued logic. 
This process merely plays a heuristic role which enables us to evoke some possible diagnostic or therapeutic constructs. It will be necessary to explore these pathways logically for a second time through search for signs which will enable the final diagnosis to be established. This should be elaborated by a request for relevant complementary tests. This reasoning sequence is designed to search for diagnostic certainty supported by reliable knowledge. Beside the evocation knowledge, the system provides expert knowledge expressed in the form of production rules for the thérapeutic or diagnostic decision.

\subsection{General Architecture of the System}

Figure 1 provides an overview of the SPHINX architecture. The user communicates with the MEDIUM [42] component, ensuring coherent dialogue throught his own knowledge and incorporating into it the basic signs and patient test results. Through the MONITOR, MEDIUM is connected with the decision module EXPERT (figure 1), which carries out the tasks on its agenda using knowledge rules. It may be said that whereas MEDIUM assures coherent dialogue, EXPERT ensures the relevance of the system discourse when in search of clinical signs or requesting para-clinical tests.

\subsection{Knowledge Representation: Entities of the Medical Discourse, Context, Heuristics and Decision Rules}

Elementary facts and concepts in current practice such as symptoms, clinical signs, examination results, syndromes, diagnoses and therapies are called entities in the system fully described by a set of attributes. The entities are represented by frames which are a restriction of the frames of Minsky [14]. This limitation rests essentially on the fact that an attribute cannot itself be a frame. Each attribute becomes a slot of the frame entity and it may be necessary to fill this slot in order to refer to other entities.

The consultation begins by activation of the EVOCATION rules which initiates an agenda of goals to weigh; for each objective the system then uses a goal-directed control structure. However, where a data-driven approach is needed to use of a small number of information items provided at a given time, such as results of paraclinical investigations, SPHINX proposes ways of modifying the agenda.

\subsection{Contexts}

A heuristic process was therefore created which limits the maximum number of acceptable hypotheses and ignores those patient's signs which are not relevant to them. Alternatively, it identifies a datum which is important for the follow-up investigations. So « coarse grain » knowledge was specified in contrast to "fine grain " knowledge expressed in the rules. This distinction allows the control structure to use « coarse grain » knowledge to determine the general context of the patient; that is, the list of diagnostic (or thérapeutic) hypotheses which should be considered before looking for more specific data to affirm or reject each one. The hypotheses are developed by reference to a number of , clinical frameworks' (figure 2) and the choice of context for a given patient is made by estimating the most probable framework; this constitutes the initial phase of the system reasoning.

\subsection{Knowledge Refering to the Contexts}

The choice of context is made at the consultation during evocation phase which is set in motion as soon as the system has enough knowledge to consider it relevant. For this, EXPERT uses knowledge of the type:

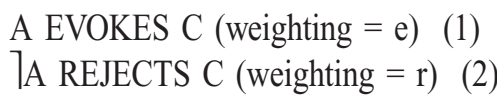

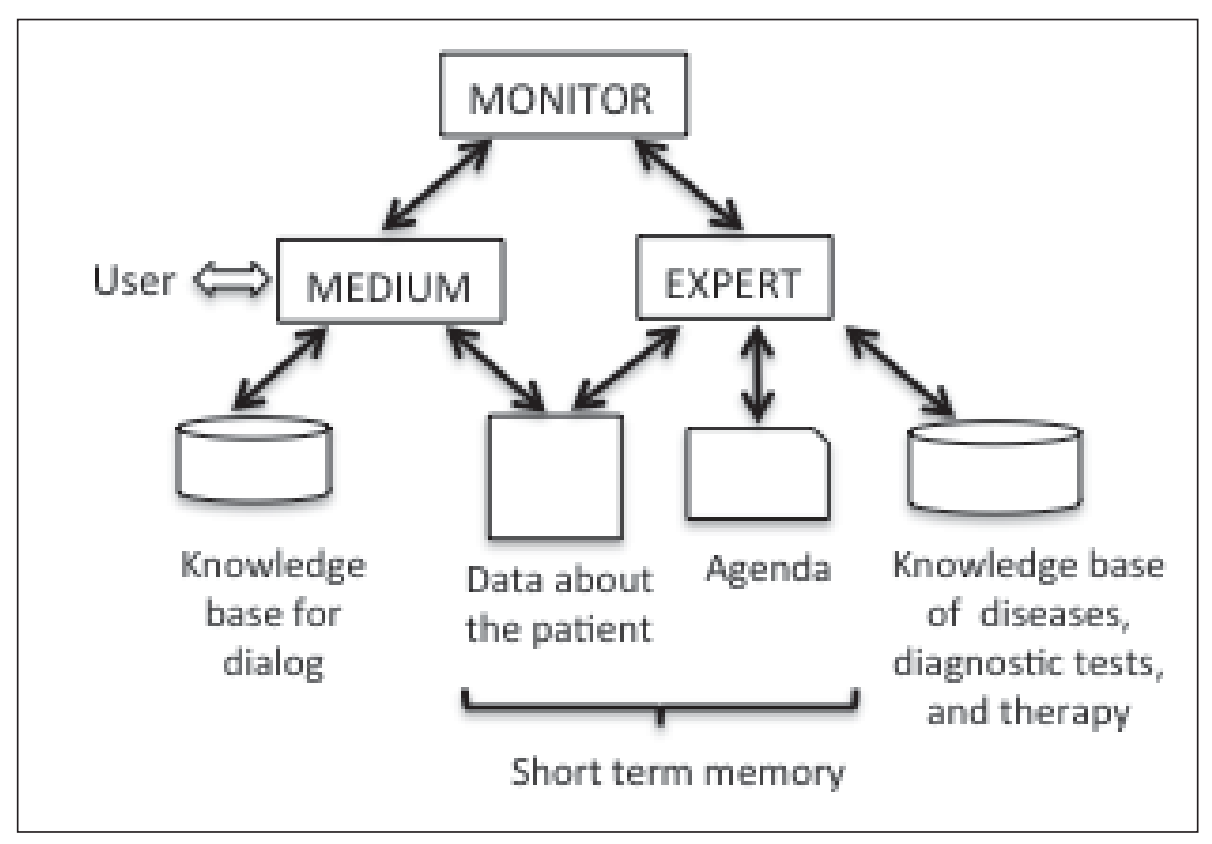

Fig. 1 General presentation of SPHINX. The components MEDIUM and EXPERT communicate through the MONITOR and together control the facts base wich contains patient data and the conclusions of EXPERT. 


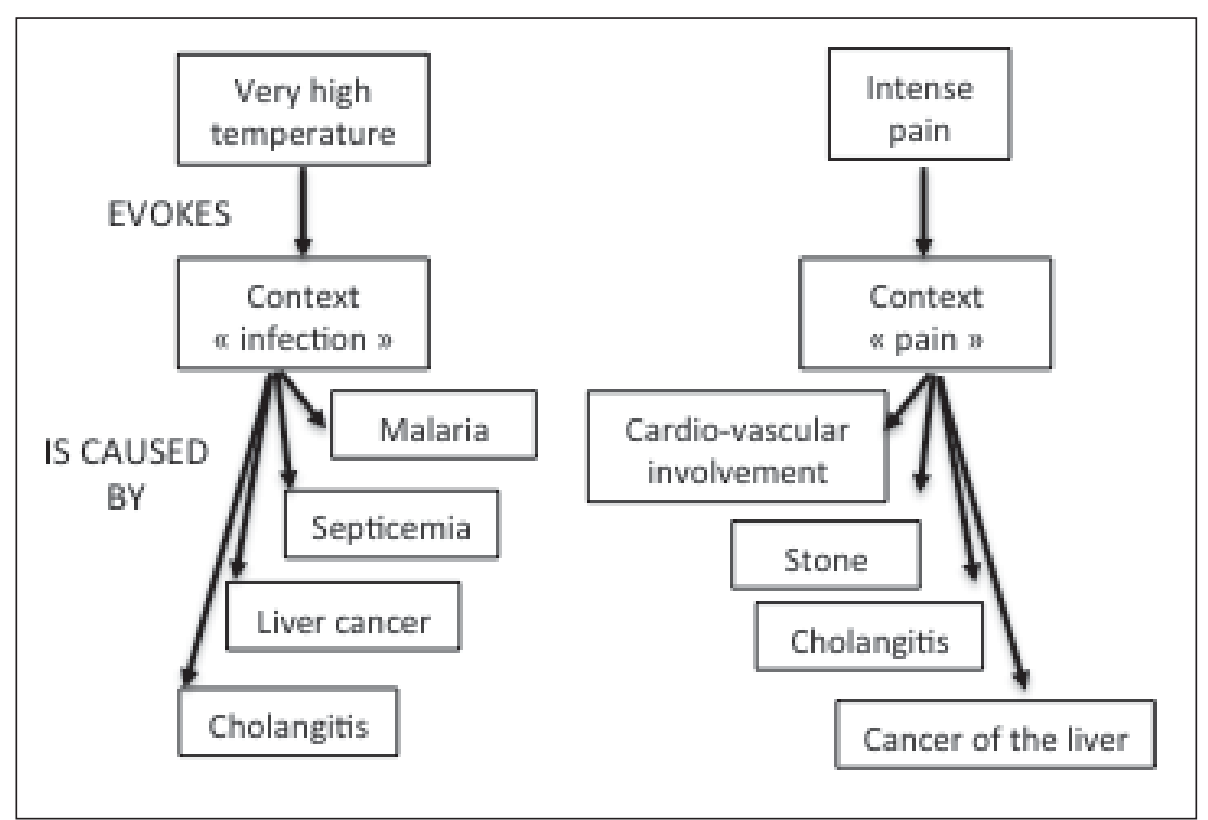

Fig. 2 Schematic concept of contexts. They enable the sumptomatology of the patient to be characterized and managed

where $\mathrm{A}$ is the expression of a typical entity with evoking power e for context $\mathrm{C}$, and (2) conveys context $\mathrm{C}$ in the absence of $\mathrm{A}$ being rejected with a weighting of r. Evocation and rejection capacities are set empirically by the expert with values between 0 and 1 .

Therefore, to identify the context or contexts closest to the clinical picture a fuzzy, pattern matching' module was created which can be activated by the evocation rules and is activated for a given patient by the first rule triggered.

Initially, the system agenda requires that the task of evocation be carried out such that the system will use the evocation rules in the order in which they appear. The first rule triggered allows evocation to proceed, which is completed with development of the agenda where diagnoses that the system will attempt to substantiate are included. In the case where the data base provided spontaneously by the consultant is inadequate, the rules lead to context definition asking for further information such as the patient's age, his state of mind, or the need for emergency (hospital) admission.

\subsection{Production Rules \\ Diagnostic or Therapeutic Knowledge Rules}

These rules enable diagnoses to be worked out or therapies proposed. The system considers them according to their associated goal, so they are triggered during backward chaining.

\section{Examination Interpretation Rules}

Some entities are amalgamated into classes and should be read in the aggregate; particularly in the case of paraclinical examinations whose general interpretation develops from elementary descriptive entities. This knowledge is expressed in the form of antecedent production rules, that is, triggered in forward chaining, which

takes into account all the components which the examination reveal. The use of such verbs as TO THINK OF, TO LOOK FOR, TO IGNORE, provide a system which is data-driven not only in the initial phase of the consultation but also when paraclinical results are interpreted (figure 3). They permit update in the agenda contents.

The MYCIN project gave rise to a number of developments in the domain of expertise transfer, through the Guidon project of W. Clancey. Our team also carried out research in this domain. An application of this type was developed in 1984, as part of the $\mathrm{PhD}$ project of Dominique Fieschi [43]. This work is just one example of the many PhD theses or research dissertations on the system environment, the validation of knowledge bases, knowledge transfer tools and more ergonomic man/machine interfaces generated by the SPHINX project at the start of the 1980s.

\subsection{Evaluation and Test in Current Practice}

The initial objective was to place SPHINX [44] among practitioners with different levels of expertise (three general practitioners and two experts); and the second objective was the estimation of the degree of expertise of the system through a study about consensus and disagreement between the system and the evaluators.

The SPHINX system agrees most frequently with at least one of the experts and with both experts together. The results implied that SPHINX had

\begin{tabular}{|ll|}
\hline R: IF & Haemoglobin low \\
& Red cells increased \\
& Microcytosis \\
& Hypochromia \\
THEN: & Pseudo microcytic polycythaemia (polyglobulism) \\
& TO IGNORE: Addison Biermer anaemia \\
& TO THINK OF: Haemoglobinopathy \\
\hline
\end{tabular}

Fig. 3 Example of a production rule in SPHINX 
a high level of expertise, lying somewhere between that of the general practitioners and that of the experts.

The use of the system was tested in the target environment for six months, by 38 general practitioners in the Provence region [45], using Minitel terminals, which were then widespread in French homes with telephones and in the workplace. The aim of the study was to determine the extent to which such a system could provide support and be both useful to, and used by, general practitioners in their daily practice. Studying principally the value of decision support in the doctor's office, we were confronted with a complex situation, with strong interactions between:

- The system itself, its user-friendly nature, its possibilities and the means of access to advice: available through Minitel, connected to the Vax of the Faculty of Medicine via the telephone network;

- The sample of participating doctors and their interest in computing (this was a very important issue in 1984-86)

- The medical domain of application and the extent to which it was of interest to the general practitioners participating in the study,

- The quality of the advice supplied.

In the application offered to doctors, we added to the expert system itself a number of additional documentary functions of relevance to the management of diabetes. The additional documents supplied involved various nutritional menus providing different numbers of calories and information about foot hygiene for diabetics, for example. We were intrigued by the success of these additional documentary functions, which did not relate to decision-making in the strict sense of the term but were very much appreciated and used by doctors in the field.

The participating doctors used the system in daily practice, during consultations at their surgeries and, in some instances, during home visits to patients. This was possible due to the widespread availability of Minitel in France at the time.

\section{The Benefits of Experience and the Development of the Team's Projects}

Was naivety in the analyses or an absence of lucidity entirely responsible for exaggerating the promise of AI? Were there not sometimes ,political“ attitudes at work, promoting AI to decision-makers and funding bodies, to obtain additional funding for the research? Amongst laymen, and certain politicians in particular, was there not a desire to hope for more than was reasonable and to dream ${ }^{2}$ ? Some people seemed to think that it would be possible to construct gigantic knowledge bases by translating phrases from medical treatises and that these systems would rapidly become available to doctors. Whatever the reality, the researchers in this domain seem to have been unable to convince people that these visions were not realistic. This situation arose from the confusion generated by the view that the performance of these systems would make them acceptable to healthcare professionals. This was clearly an error of judgment but not something inherent to the AI approach.

The research carried out during these years did not lead to the rapid adoption of these systems in everyday practice. Such research was necessary to establish the performance of ambitious computerized decision support systems but was not sufficient to achieve the objective of the generalized use of such systems in practice. The development

\footnotetext{
2 I remember a meeting at Marseilles in 1984 attended by French politicians in office, who expected and demanded concrete results by 1986 . I found it very difficult to make them understand that this timetable was not realistic. That was not what the politicians wanted to hear.
}

of consulting systems independent of information systems and without integration into the process could never hope to achieve such an objective, whatever the methodology used. We needed to recognize the slow pace of progress and to identify and understand the prerequisites and conditions for a more rapid spread of decision support systems. Integration is a widely recognized criterion of acceptability, but it may be achieved in various ways. There is no clear consensus concerning the best way to achieve this integration into the flow of healthcare practice to meet expectations concerning interactions with the doctors, patients and healthcare professionals involved in this process.

We should remember that AI approaches and methods have made a rich contribution. The questions concerning decision mechanisms and knowledge representations have been determinants of progress in the design of computerized systems for use by healthcare professionals. The general methods defined are still widely used. In a revised form, they are currently used in projects in which the knowledge used is no longer based on expert opinion, but obtained from good practice guidelines. The issue is no longer to demonstrate that a computer can perform as well as an expert in consultation, but to provide effective assistance to the doctor and to evaluate the added value provided by the computerized system. The debate about whether machines might one day replace humans has moved on. A change in mentality has also contributed to the search for complementarity between humans and machines and for tools that are more appropriate and better integrated into the management process. The conversion of all documents into a digital format, generalized modes of production in all sectors of activity, possibilities for data treatment and transmission, the appropriation of treatment devices and access to information, the development of the Internet and changes in functions, particularly those of a documentary nature, have considerably modified the technologi- 
cal, educational, behavioral and sociological context of the various actors.

These approaches to decision support have also been called into question, in line with developments in other domains:

- The development of structured clinical protocols to guide practice. This was carried out at Beth Israel Hospital in Boston, in particular, beginning in the early 1970 s, for outpatient practices [46].

- The development, at the end of the 1980 s and the beginning of the 1990 s, of formal methods for the specification of good clinical practice guidelines [47], and to specify and manage the workflow in a clinical environment [48].

Many researchers thus began to study the interaction with the doctor during the care process, through intelligent interfaces and appropriate computer physician order entry (CPOE) systems. A need to integrate decision support tools, regardless of the methods used, into broader information systems responding to the pratical routine of professionals became evident. This need appeared clearly in the pioneering studies of the team from LDS Hospital in Salt Lake City, whose work with the HELP system [49] rapidly opened up this avenue of research. The team of $\mathrm{C}$. MacDonald [50] at Regenstrief Hospital, with reminders, also worked in this area. Note that organizational dimensions, the role of actors and the contribution of social sciences, were well outside the field of studies in the 1970s and 1980s. Projects tended to be academic prototype studies implemented outside the hospital information system. Process analyses shed light on the way in which knowledge and attitudes are developed and shared, improving our understanding of these aspects.

From the second half of the 1980s onwards, our team focused on the architecture of information systems and the reconciliation of heterogeneous data, which are often encountered in practice. Addressing these questions, which are less theoretical than decision models, should facilitate the integration of decision support applications into the workflow of healthcare professionals. This multiform support has been revised and today takes diverse forms. Practice support supplies reliable and relevant data at the right moment and access to relevant contextualized documents, and it facilitates communication with the healthcare professionals involved in patient management, the issuing of alerts, warnings or reminders of good practice or expert advice. All these functions must be performed by software components integrated into information systems guiding the management of care processes. This approach should greatly attenuate the initial errors of judgement concerning the performance and acceptability of these systems. Our research mostly addresses questions relating to the structuring, organization and presentation of information to ensure the interoperability of these information systems and documents, searches for information in heterogeneous databases, the integration of good practice guidelines into medical processes and the various information subsystems, and the adaptation of CPOEs. These areas of research have become important, together with definition of the semantic references associated with them. Publications from this period demonstrate the increasing importance accorded by medical informatics researchers to these subjects and corollary normative issues. In addition, these architectures required the implementation of standards, one of the strengths of which is the possibility for sharing and reusing information and knowledge. Substantial efforts have been made for standardization in the domain of decision support systems since the definition of the Arden Syntax [51] and standardized exchange formats for guidelines towards the end of the 1990s. These questions were outside the domain of our research on the applications of AI in medicine a decade earlier. They have become more important but, in attempting to resolve them, researchers are increasingly having to make way for IT engineers. In addition, integration into hospital information systems involves negotiation with hospital managers, who must be convinced of the solid basis and importance of these information systems. These debates are frequently focused on billing and regulatory quality assurance and administrative functions. Consequently, researchers have to spend considerable amounts of time becoming negotiators and opinion leaders in their healthcare structures if they are to effectively incorporate decision support functionalities in routine clinical use - whether or not these are inspired by AI approaches.

\section{References}

1. Ledley RS, Lusted L. Reasoning foundations of médical diagnosis. Science 1959;130:9-21.

2. Warner HR, Toronto HF Veasy LG A mathematical approach to médical diagnosises : Applications to congénital heart disease. JAMA 1961;177:177-83.

3. Gorry GA, Barnett GO. Experience with a model of sequential diagnosis. Comput Biomed Res 1968:490-507.

4. De Dombal FT, Leaper DJ, Staniland JP, McCann AP, Horrocks JC. Computer Aided Diagnosis of Acute Abdominal Pain. BMJ 1972;2:9-13.

5. Deutsch T, Carson E, Ludwig E. Dealing with Medical Knowledge: Computers in Clinical Decision Making. New York: Plenum press; 1994.

6. Davis R, King JJ. The Origin of Rule-Based Systems in AI. In: Buchanan BG, Shortliffe EH, editors. Rule-Based Expert Systems. The Mycin experiments of the Stanford Heuristic Programming Project. Addisson-Wesley; 1984. p. 20-52.

7. Newell A. Production systems: Models of control structures. In: Chase WG, editor. Visual Information Processing. New York: Academic Press; 1973. p. 463-526.

8. Shortliffe EH. Computer-Based Medcial Consultations: MYCIN. New York: Elsevier; 1976.

9. Institute of Medicine. To Err is Human: Building A Safer Health System. Consensus report. New York: National Academy Press; Nov. 1999.

10. Institute of Medicine: Crossing the Quality Chasm: A New Health System for the 21st Century Consensus report. New York: National Academy Press; March 2001

11. Ganascia JG. L'âme-machine. Paris: Seuil; 1990.

12. Newell A, Simon H. GPS a Program that Simulates Human Thought. In: Feigenbaum EA, Feldman J, editors. Computers and thought. New York: McGraw-Hill; 1963.

13. Winograd T. Frame representations and the procedure/declarative controversy. In: Bobrow DG, 
Collins A. Representation and the Understanding, Studies in Cognitive Science. New York: Academic Press; 1975. p. 185-210.

14. Minsky M. A Framework for Representing Knowledge. In: Winston PH, editor. The psychology of Computer Vision. New York: McGraw-Hill; 1975. p. 211-77.

15. Pauker SG, Szolovits P. Analyzing and simulating taking the history of the oresent illness: context formation. In: Schneider W, Sgvall-Hein AL, editors. IFIP Working Conference on Computationa Linguistics in Medicine. Amsterdam: North Holland; 1977. p. 109-18.

16. Fagan L, Shortliffe EH, Buchanan BG. ComputerBased Medical Decision Making: from MYCIN to VM. Stanford Heuristic Programme Project, HPP79-18; 1979.

17. Clancey WJ. The Epistemology of a Rule-based Expert System: A Framework for Explanation. Artificial Intelligence 1983;20:215-51.

18. Patil RS. Causal Representation of Patient Illness for Electrolyte anda Acid-Base Diagnosis. PhD Thesis. MIT Laboratory for Computer Science. Cambridge Mass; 1981.

19. Kulikowski CA, Weiss SM. Representation of Expert Knowledge for consultation: the CASNET and expert Projects. In: Szolovits P. Artificial Intelligence in Medicine. Westview, Colorado; 1982. p. 21-55.

20. Pople HE Jr. Heuristic Methods for imposing Structure on Ill Structured Problems: The Structuring of Medical Diagnostics. In: Szolovits P. Artificial Intelligence in Medicine. Westview, Colorado; 1982. p. 119-90.

21. Miller PL. Medical Plan-Analysis by Computer. In: Van Bemmel JJ, Ball MJ, Wigertz O, editors. MEDINFO 83. Amsterdam: North Holland;1982.

22. Rector AL. Defaults, exceptions and ambiguity in a medical knowledge representation system. Med Inform Oct-Dec 1986;11(4):295-306.

23. Zarkadakis G, Carson ER, Cramp DG, Finkelstein L. ANABEL: intelligent blood-gas analysis in the intensive care unit. Int J Clin Monit Comput 1989;6(3):167-71

24. Fox J, Glowinski A, Gordon C, Hajnal S, O’Neil M. Logic engineering for knowledge engineering: design and implementation of the Oxford System of Medicine. Artif Intell Med 1990;2:323-39.

25. Timpka T, Hjerppe R, Strömberg D, Möller I, Bjurulf P, Gill H, et al. Decision support for General Practitioners by integrating paradigms: Hypertext, Knowledge based and Electronic library. In: Salamon R, Blum B, Jörgensen M, editors.
MEDINFO 86. Amsterdam: North-Holland; 1986. p 96-100.

26. Adlassnig KP, Kolarz G. Representation and semiautomatic acquisition of medical knowledge in CADIAG-1 and CADIAG-2. Comput Biomed Res 1986;19(1):63-79.

27. Lesmo L, Marzuoli M, Molino G, Torasso P. An expert system for the evaluation of liver functional assessment. J Med Syst 1984;8(1-2):87-101.

28. Quaglini S, Stefanelli M, Barosi G, Berzuini A. ANEMIA: an expert consultation system. Comput Biomed Res 1986;19(1):13-27.

29. Weiss S Kulikowski CA. EXPERT: a system for developing consultation models. Proc Sixth Int Conf on Artif Intelligence, Tokyo; 1979.

30. Gascuel O. SAM: un système expert dans le domaine médical. [An expert system in the medical domain] PhD Thesis, Université de Paris VI; 1981.

31. Fontaine P, Beuscart R, Lecoq P, Vambergue A. DIABAID : Un système expert d'aide à l'adaptation de l'insulinothérapie. [An expert system helping for adaptation of insulinotherapy]. Innovation et Technologie en biologie et médecine 1997;18(6):421-31

32. Sanchez E, Soula G. Possibilistic analysis of fuzzy modelling in medecine. In: Vantenkiste, Young, editors. Modeling and data analysis in biotechnology and medical engineering. Amsterdam: North Holland;1982. p.127-38.

33. Gouvernet J, Caraboeuf M, Ayme S. GENDIAG: a computer-assisted facility in medical genetics based on belief functions. Methods Inf Med 1985;24(4):177-80

34. Soula G, Vialettes B, San Marco J.JL. PROTIS: a fuzzy deduction rule system: application to the treatment of diabetes. Van Bemmel JH, Ball MJ, Wigertz O, editors. Medinfo 1983. Amsterdam: North Holland; $1983 ;: 533-6$.

35. Lanzola G, Quaglini S, Stefanelli M. Knowledgeacquisition tools for medcial knowledge-based systems. Methods Inf Med 1995;34(1-2):25-39.

36. Fieschi M. Intelligence Artificielle en Médecine : des systèmes experts. Paris: Masson; 1984

37. Fieschi M. Artificial Intelligence in Medicine-Expert systems. Translated by D. Cramp. London: Chapman and Hall; 1990

38. Fieschi M, Joubert M, Fieschi D, Botti G, Roux M. A program for expert diagnosis and therapeutic decision. Med Inform (Lond) 1983;8(2):127-35.

39. Rescher N. Many Valued Logic. NewYork: McGrawHill; 1969.

40. Shafer G. A Mathematical Theory of Evidence. Princeton University Press; 1976.
41. Soula G. Aide à la décision en logique floue. Application en médecine. Thèse Doctorat de Biologie Humaine. [Decision support in fuzzy logic. Doctora thesis] Marseille; 1981

42. Joubert M, Fieschi M, Fieschi D, Roux M Knowledge representation and utilisation in a manmachine dialogue with a medical decision aid system. Methods Inf Med 1982;21(2):59-64.

43. Fieschi D. Contribution au système expert SPHINX Application à l'enseignement médical. Thèse de $3^{\text {eme }}$ cycle. [Contribtion to the SPHINX expert system: Application to medical teaching] Universite Paris 6; 1984.

44. Michel C, Botti G, Fieschi M, Joubert M, San Marco JL, Casanova P. Validation of a Knowledge Base Intended for General Practitioners to Assist Treatmlent of Diabetes: A blind Study. In: Salamon R, Blim BI, Jorgensen M, editors. Proc. Medinfo 86. Amsterdam: North Holland; 1986. p. 122-6.

45. Fieschi M, Botti G, Michel C, Joubert M, Proudhon H, Fieschi D. Etude de mise à disposition des praticiens généralistes d'un système expert d'aide à a thérapeutique du diabète (étude de faisabilité). Rapport de fin de contrat ADI n ${ }^{\circ}$ 84-747 Faculté de Médecine de Marseille.

46. Komaroff Al, Black WL, Flatley M, Knopp RH, Reiffen B, Sherman H. Protocols for physicians assistants. Management of Diabetes and Hypertension. N Engl J Med 1974;290(6):307-12.

47. Abendroth TW, Greenes RA. Computer presentation of clinical algorithms MD Comput 1989;6(5):295-9.

48. Ciccarese P, Caffi E, Quaglini S, Stefanelli M Architectures and Tools for innovative Health Information Systems : The Guide Projetc. Int J Med Inform 2005;74(7-8): 553-62.

49. Pryor TA, Gardner RM, Clayton PD, Warner HR The HELP System. J Med Syst 1983;7:87-102.

50. McDonald CJ. Protocol-based computers reminders, the quality of care and the non-perfectability of man. N Engl J Med 1976;295:1351-5.

51. Hripcsak G, Wigertz OB, Kahn MG, Clayton PD, Pryor TA. ASTM E31.15 on health knowledge representation : the Arden Syntax. Stud Health Technol Inform 1993;6:105-12.

Correspondence to:

Professor Marius Fieschi

16 Boulevard de Haguenau

13012 Marseille

France 